\title{
LOCALIZATION USING FILTERED DGPS
}

\author{
Arun Kumar G C $\mathbf{C}^{1}$, Varalakshmi K R $\mathbf{R}^{2}$ \\ ${ }^{1}$ Asst Prof, Dept of ECE - SVIT (affiliated to VTU - Belgaum), Bangalore, Karnataka, India \\ ${ }^{2}$ Asst Prof, Dept of ECE - SVIT (affiliated to VTU - Belgaum), Bangalore, Karnataka, India
}

\begin{abstract}
In this paper, localization of the robot is achieved by considering two Global Positioning Systems (GPS) or DGPS. Differential Global Positioning System (DGPS) is interfaced with MBED with the help of Zigbee protocol. For accurate localization of mobile robot DGPS is preferred. Filters are used to remove the erroneous noise from the data obtained from GPS. Low pass IIR filter for $D G P S$ is realized. The project work discusses each of these approaches for localization in Outdoor environment. The above algorithm is implemented on MBED Platform. Simulation results are extracted using Matlab.
\end{abstract}

\section{Keywords—localization, Outdoor environment, Low pass IIR filter, DGPS, MBED}

\section{INTRODUCTION}

GPS are used to estimate localization with respect to earth's latitude, longitude and altitude axis. In earlier days Researchers used to manually feed the localization data for the robot. Later after the invention of GPS, Researchers made use of GPS data in order to estimate localization [1] [8]. The GPS all alone is of less accurate and is prone to noise. Since there is random fluctuation in localization update, later the researchers have came up with an idea of DGPS, in which two GPS devices were used together. First GPS sensor was placed on ground point (reference point) and is interfaced with first MBED and the second GPS was placed on robot and is interfaced with second MBED. For communication between the two GPS, Zigbee (XBEE Company) protocol is preferred [2].

Platform - Embedded Platform

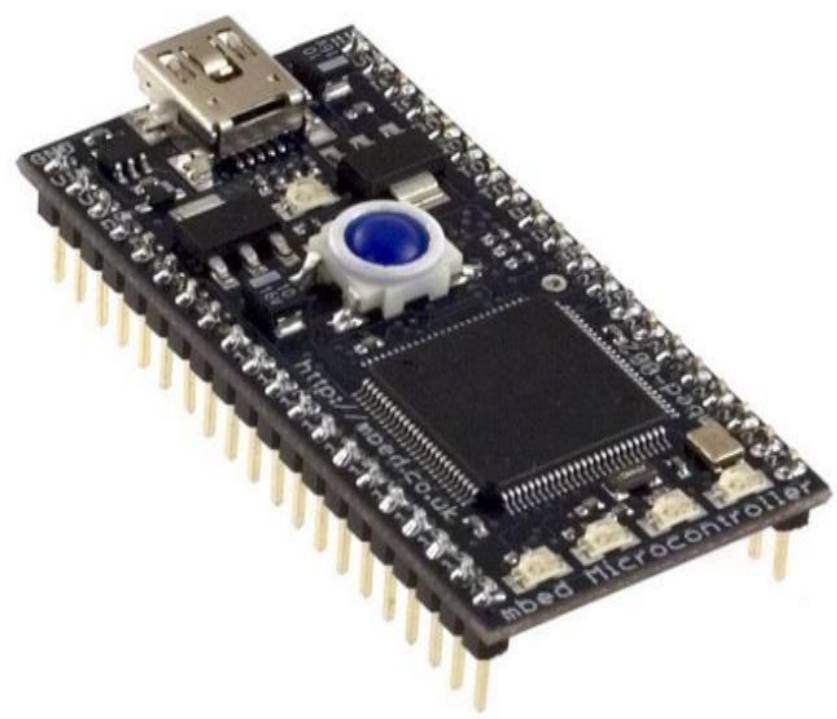

Fig 1 MBED ARM Cortex-M3 LPC1768
MBED LPC1768 ARM Cortex-M3 is preferred as a controller because of its high performance, low power consumption. It supports $\mathrm{I}^{2} \mathrm{C}$, SPI, USB, CAN, Ethernet; online compiling makes the programming task much easy. GPS module is connected to ARM Micro Controller MBED [1]. Researchers have come up with many ideas for estimating localization and many has been proposed in the field of research in robotics. In earlier days for estimating localization Ultrasonic positioning systems and infrared network systems which are available at low cost, small were used which can be easily interfaced with the controller.

However, if the target is situated far, ultrasonic sensor fails to detect the object; hence those kinds of systems were not preferred. Due to large signal interference it is not preferred nowadays. Later they have come up with an idea of Radio Frequency Identification (RFID), which requires additional equipment which was tied on robot; RFID requires high cost to implement and hence it is also not preferred. For Indoor navigation Global Positioning System (GPS) cannot be preferred, as it requires Line of Sight (LoS) with the satellites and has a slow update rate of one second approximately. So, the researchers have come up with a new idea of using Differential GPS which has an accuracy level close to few tens of centimeter and are available and used in commercial purpose and in vehicles also which makes them attractive for localization of outdoor mobile robots navigation [1] [2] [4].

Figure 2 describes the project flow in which the project is executed. GPS is connected to MBED; GPS device requires power supply and is powered by MBED micro controller. Tx and Rx pins of MBED are connected to Rx and Tx pins of GPS for serial communication. As GPS data is prone to noise, accuracy is in terms of few hundreds of meters. Hence DGPS data is preferred which has a accuracy level of few centimeters. On the application point of view robots should navigate to destination accurately, filters are preferred basically first or second order filter. For simplicity first order is chosen. 
As two micro controllers are not wired, Zigbee protocol is preferred for wireless communication between the two $\mu \mathrm{C}$.

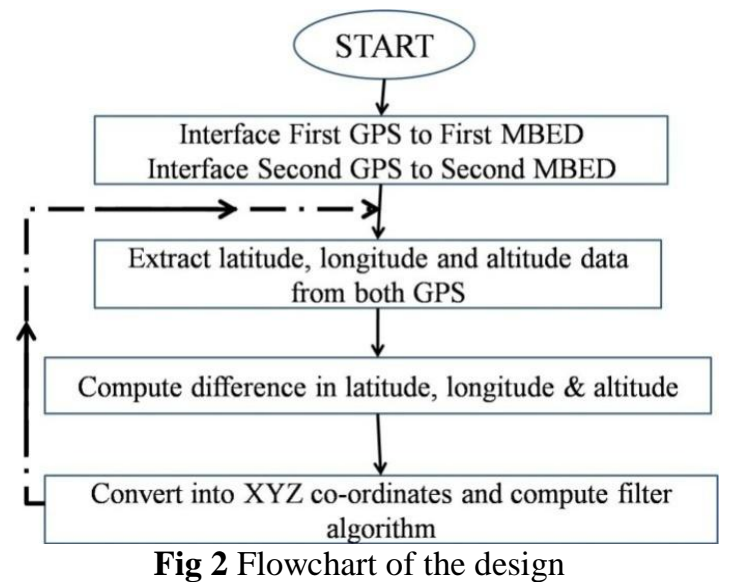

Figure 2 describes the design flow of the paper. First GPS devices are connected with MBEDs and the communication between two MBED is done using Zigbee protocol (XBEECompany). The data obtained from GPS is erroneous to noise, so appropriate filter algorithm is chosen according to the design constraints.

\section{FILTER ALGORITHM DESIGN}

\subsection{IIR Low Pass Filter}

Infinite Impulse Response (IIR) is used to suppress or eliminate the unwanted rapid fluctuation of GPS data. The complexity of the system directly depends on the order of the system. Gain ' $\beta$ ' of the system decides the quantity of noise to be removed from noise and ' $\beta$ ' decides the percentage of present data to be considered and (1- $\beta$ ) determines the fraction of past data to be considered.

$$
0<\beta<1
$$

Output of the low pass Infinite Impulse Response (IIR) filter equation for the first order system can be written as:

$$
b[i]=(1-\beta) * b[i-1]+\beta *(c[i]-b[i-1])
$$

$$
\begin{array}{ll}
\text { where } & \\
\mathrm{c}[\mathrm{i}] & =\text { present input } \\
\mathrm{b}[\mathrm{i}] & =\text { present output } \\
\mathrm{b}[\mathrm{i}-1] & =\text { previous output } \\
\mathrm{a} & =\text { gain parameter }
\end{array}
$$

GPS data will provide information regarding latitude (lat), longitude (long) and altitude (alt), N/S direction and also E/W direction information. Localization of reference point can be estimated by using set of eqns. of eqn. $2 a$, eqn. $2 b \&$ eqn. $2 \mathrm{c}$ from GPS sensor.

Similarly Localization of mobile robot with respect to reference point can be estimated by using set of eqns. of eqn. $3 a$, eqn. $3 b$ \& eqn. $3 c$ from GPS sensor.
Set of eqns. of 4 determines the gain quantity which is dependent on both input and output. ' $\beta$ ' on input and ' $1-\beta$ ' on output.

From first GPS sensor extracting only latitude, longitude and altitude information, these values are converted into $\mathrm{XYZ}$ coordinates and is given by,

$$
\begin{aligned}
& \mathrm{X} 1=\mathrm{R} * \cos (\text { lat } 1) * \cos (\text { long } 1) \\
& \mathrm{Y} 1=\mathrm{R} * \cos (\text { lat } 1) * \sin (\text { long } 1) \\
& \mathrm{Z} 1=\mathrm{R} * \sin (\text { alt } 1)
\end{aligned}
$$

From second GPS extract latitude, longitude and altitude information. Converting into coordinates:

$$
\begin{aligned}
& \mathrm{X} 2=\mathrm{R} * \cos (\text { lat } 2) * \cos (\text { long } 2) \\
& \mathrm{Y} 2=\mathrm{R} * \cos (\text { lat } 2) * \sin (\text { long} 2) \\
& \mathrm{Z} 2=\mathrm{R} * \sin (\text { alt } 2)
\end{aligned}
$$

First MBED sends GPS data (latitude, longitude and altitude) into second MBED via Zigbee protocol

Since DGPS is used for calculating the localized coordinate's difference of second GPS coordinates with the first is taken. Localization of mobile robot considering localization estimate information from set of eqn. 2 and eqn. 3 with respect to ground point and is given by,

$$
\begin{aligned}
& € X=X 2-X 1 \\
& € Y=Y 2-Y 1 \\
& € Z=Z 2-Z 1
\end{aligned}
$$

For serial communication between GPS devices and MBED micro controller, BAUD rate was chosen to be 9600 . If lock is enabled, localized data is fetched from the satellite by GPS sensors.

\section{IMPLEMENTATION DESIGN}

First GPS senses the north pole of earth (Heading measurement). GPS sensor receives around 5 sets of data stream (\$GPGSV, \$GPGGA, \$GPGSA, \$GPVTG \$GPRMC). For the application point of view \$GPGGA is chosen. As \$GPGGA data stream provides information regarding latitude, altitude, longitude information and it also provides information regarding E/W direction and N/S direction. [1] [3].

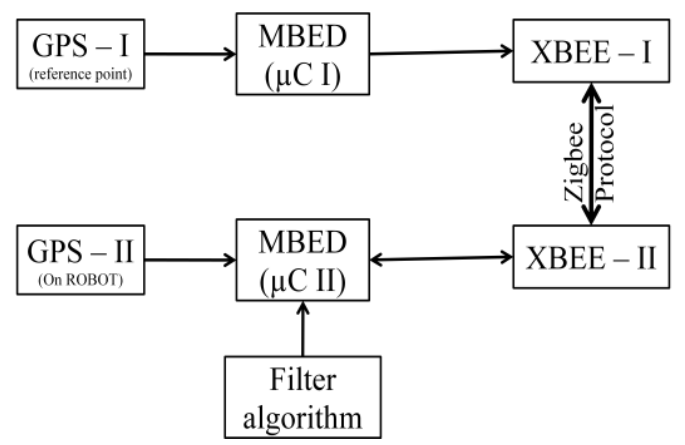

Fig 3 Block diagram representing communication between two MBED's 
By which localization of robot can be estimated using earth's coordinates in terms of $\mathrm{X}, \mathrm{Y}, \mathrm{Z}$ and the obtained data is fed to low pass IIR design. Since data obtained from As GPS sensor is prone to noise and accuracy is very much poor, Differential GPS (DGPS) is chosen or preferred.

From figure.3, two GPS devices are connected to two different micro controllers, so for communicating between two microcontrollers Zigbee protocol (XBEE Company) which supports wireless communication is chosen (preferred).

\section{SIMULATION RESULTS}

Matlab tool is used to extract Simulation results. Mobile robot is navigated in outdoor environment and localization points are plotted with the help of Matlab tool and are simulated to obtain the plots.

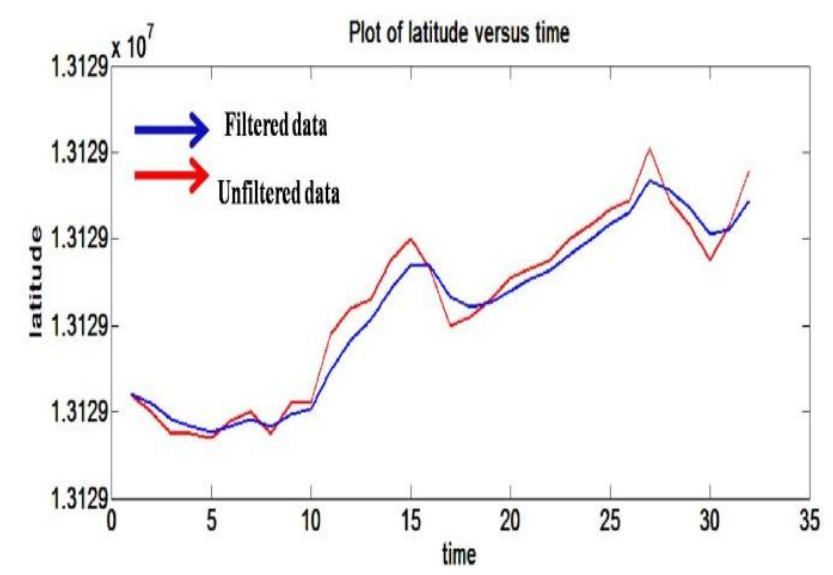

Fig 4 Filtering of latitude data obtained from GPS

Figure 4 describes the latitude variation with respect to time. When the robot is navigating towards the destination, due to rapid fluctuation in localization, in order to suppress rapid fluctuation of the data accomplished with error signal, filters are used.

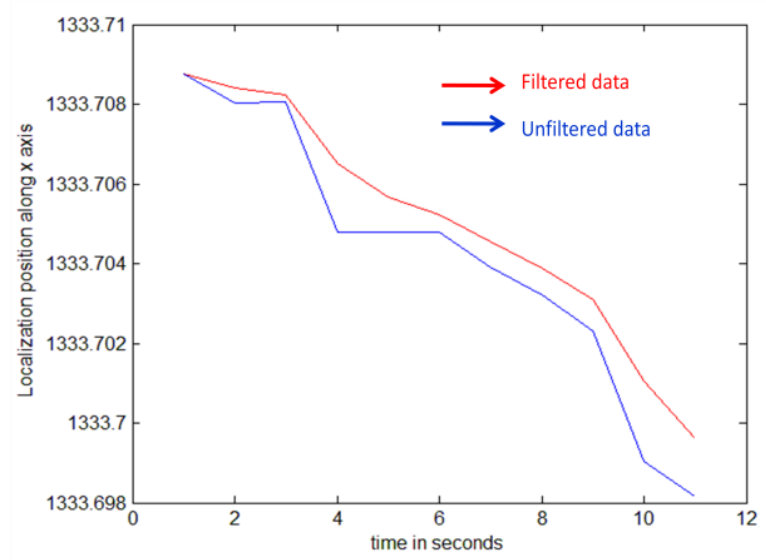

Fig 5 Localization along $x$ axis, $\beta=0.5$
Figure 5 describes the localized data along $X$ axis (latitude variation) and figure 6 describes longitude variation along $\mathrm{Y}$ axis. Filter algorithm is done in order to smoothen the waveform or to suppress rapid fluctuation in localized data.

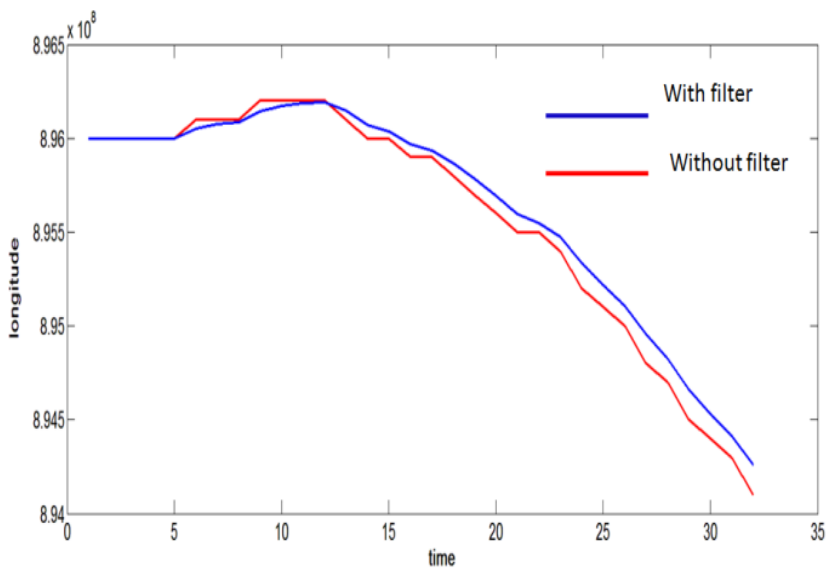

Fig 6 Filtering of altitude data obtained from GPS

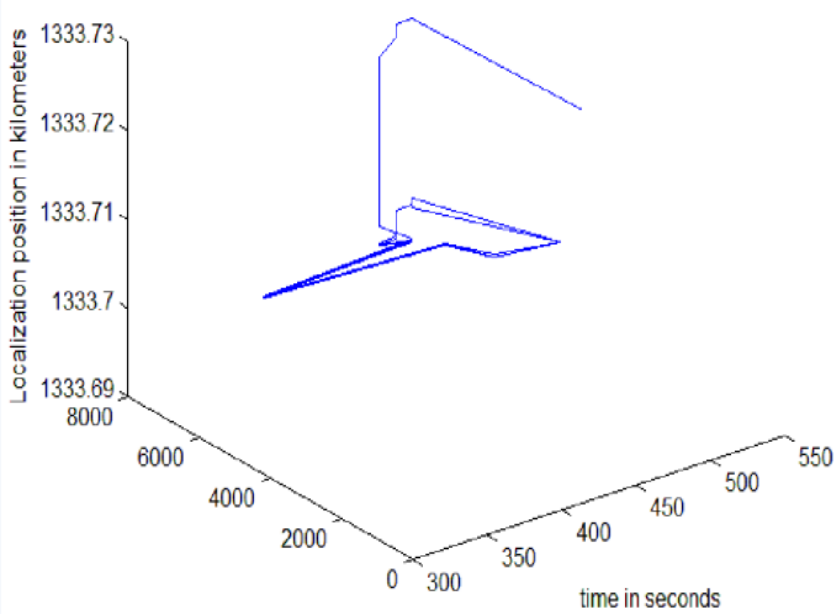

Fig 7 Localization along XYZ axis versus time (3D plot)

Figure.7 describes the localization estimate along $\mathrm{X}$ axis, $\mathrm{Y}$ axis and $\mathrm{Z}$ axis with and without filter. Gain ' $\beta$ ' was chosen to be 0.5 . (1- $\beta)$ is the fraction of quantity depending on past output, filtered response is plotted in figure 8.Gain $(\beta)$ is the fraction of quantity depending on input. 


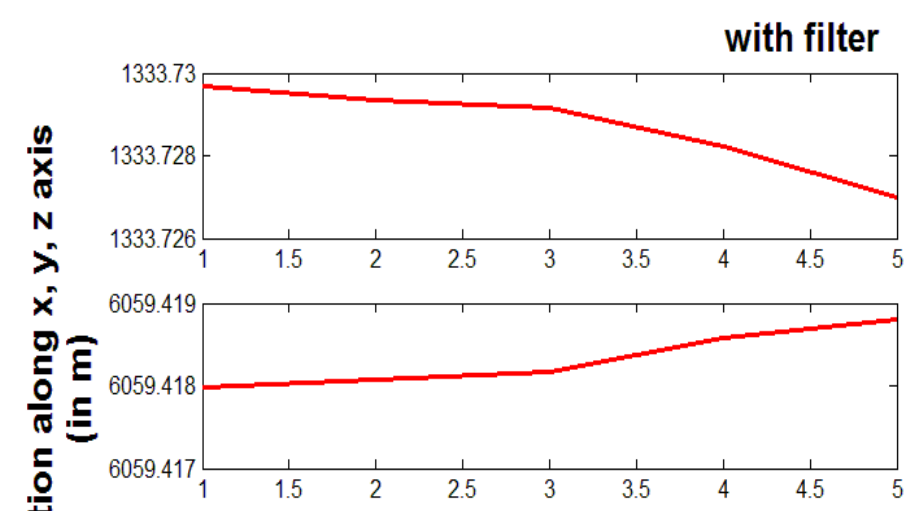

and
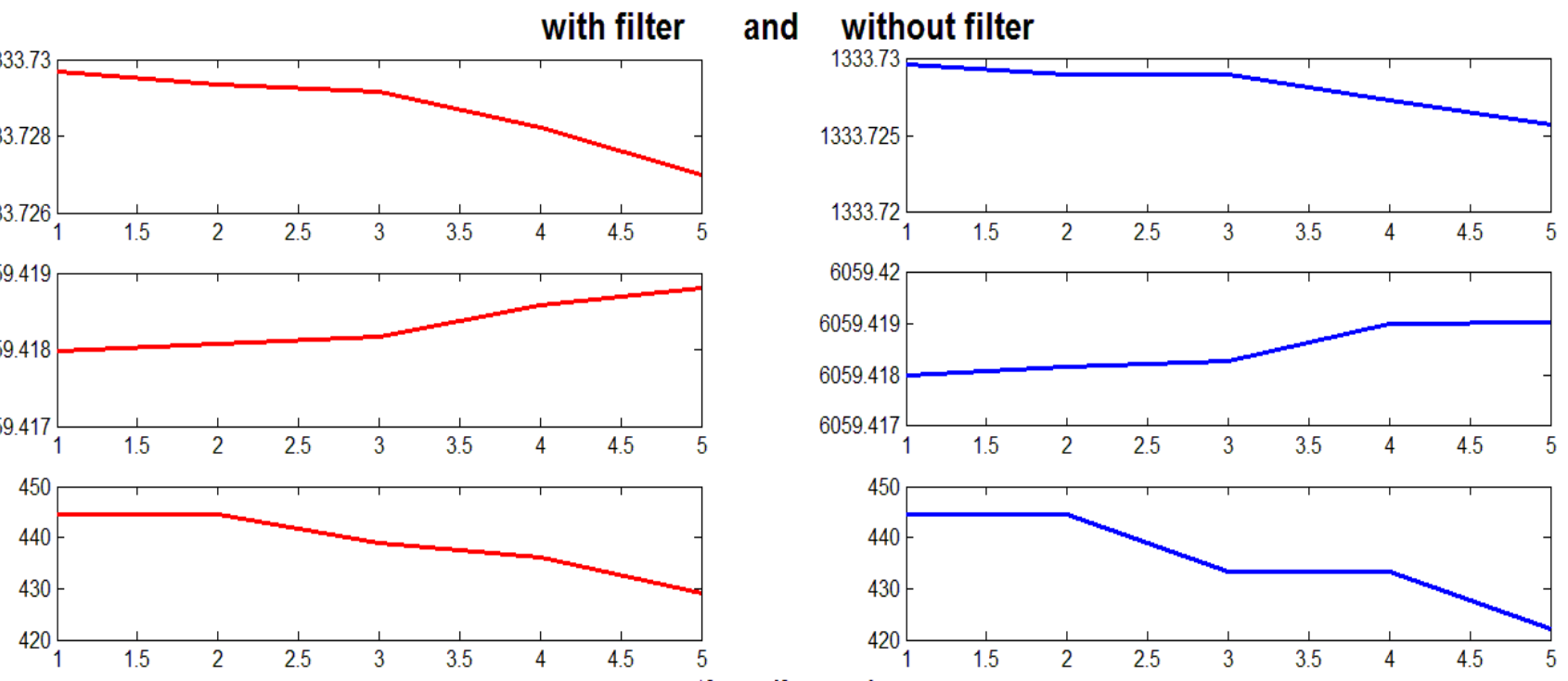

time (in sec)

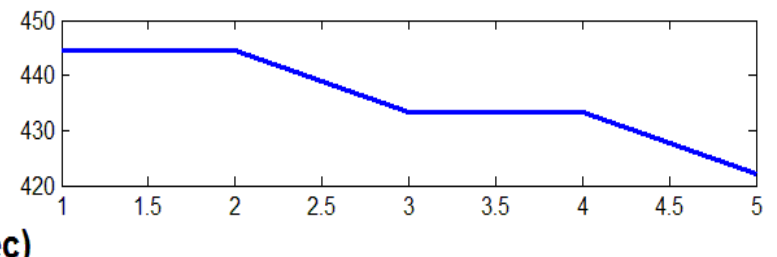

Fig 8 Localization data with and without filter

\section{CONCLUSION AND FUTURE WORK}

The proposed filter algorithm for DGPS offers fast and accurate localization in terms of earth co-ordinates and to improve the localization and navigation of mobile robot in outdoor environment or uneven surfaces accurately, multisensor (camera, pressure sensor, IMU) sensor data can be used. For Indoor navigation and in the absence of LoS, IMU and Odometry data can be considered.

\section{ACKNOWLEDGEMENTS}

The authors express their sincere gratitude to Prof. M R Holla - Director, Dr. Ramesh Babu H S - Principal, Prof. Y Jayasimha - Dean Academics, Dr K Narayan - Dean R\&D, Dr. Chitra Kiran N - HOD, Dept of ECE, SVIT. The authors would like to thank the faculties those who have supported directly or indirectly in carrying out the research work that was carried out in SVIT. The authors would also wish to thank their family members for constant encouragement and their support.

\section{REFERENCES}

[1] Arun Kumar G C, Chandrappa, Anil Kumar G C, "Real time filter and fusion of multi-sensor for localization of mobile robot", IJECET, Volume 6, Issue 1, January 2015, India, pp. 49 - 57

[2] Alejandro J. Weinstein, Kevin L. Moore, "Pose Estimation of Ackerman Steering Vehicles for Outdoors Autonomous Navigation" Golden, CO, 2010

[3] Eric North, Jacques Georgy, "Improved Inertial/ Odometry/ GPS positioning of wheeled robots even in GPS denied Environments, 2012

[4] Armando AlvesNeto, Douglas Guimaraes Macharet, "Adaptive complementary filtering algorithm for mobile robot localization", 2009
[5] Aeronautical Telecommunications, Annex 10 to the Conversion on International Civil Aviation, 1985

[6] R. Hatch K P Schwarz and G Lachapelle, "Kinematic systems in geodesy, surveying and remote sensing", pp.299 -308 1990

[7] M. S. Braasch "Improved Modeling of GPS Selective Availability", Institute of Navigation 1993 National Technical Meeting, 1993

[8] Samadzadegan, FAbdi, "Autonomous navigation of Unmanned Aerial Vehicles based on multi sensor data fusion", 20th Iranian Conference on Electrical Engineering (ICEE), pp. 868 - 873, 2012

[9] H. Wen, P. Y. Huang, J. Dyer, A. Archinal and J. Fagan, "Countermeasures for GPS signal spoofing", Proc. of the $18^{\text {th }}$ International Technical Meeting of the Satellite Division of The Institute of Navigation, pp. 1285-1290, 2005.

[10] P. Misra, P. Enge, "Global Positioning System, Signal Measurements and Performance" 2ed, Ganga-Jamura Press, Lincoln, 2006

\section{BIOGRAPHIES}

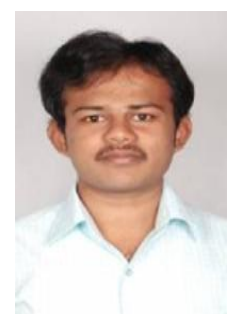

Arun Kumar G C, Working as an Assistant Professor in SVIT, Bangalore. $\mathrm{He}$ has finished his M.Tech (VLSI Design and Embedded Systems) from NMIT - Bangalore. He was also a sponsored student from TEQIP during his post graduation. He has worked in Centre of Research in Robotics for 18 months during his post graduation. He has completed his graduation (B.E) from SVIT - Bangalore. He has won second place for Mobile based car parking for SKYBUS in International level competition held at Avadi, Chennai in 2012 during his graduation. His area of interest includes Robotics, VLSI, Embedded System Design / Development. 
Varalakshmi K R, Working as an Assistant Professor in SVIT, Bangalore. She has finished her M.Tech (Digital Communication and Networking) from SJCIT, Chikkabalapura. She has completed her graduation (B.E) from NCET, Bangalore. She is having a teaching experience of 5 years. Her area of interest includes Embedded System design, Development, Networking. She has now registered for PhD in VTU university. 